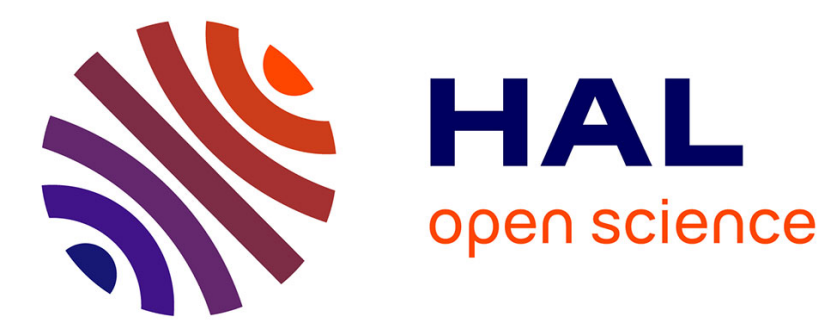

\title{
Revisiting galvanomagnetic effects in conducting ferromagnets
}

\author{
Richard Walter, M Viret, Surendra Singh, L Bellaiche
}

\section{To cite this version:}

Richard Walter, M Viret, Surendra Singh, L Bellaiche. Revisiting galvanomagnetic effects in conducting ferromagnets. Journal of Physics: Condensed Matter, 2014, 26 (43), pp.432201. 10.1088/09538984/26/43/432201 . cea-01377256

\section{HAL Id: cea-01377256 https://hal-cea.archives-ouvertes.fr/cea-01377256}

Submitted on 6 Oct 2016

HAL is a multi-disciplinary open access archive for the deposit and dissemination of scientific research documents, whether they are published or not. The documents may come from teaching and research institutions in France or abroad, or from public or private research centers.
L'archive ouverte pluridisciplinaire HAL, est destinée au dépôt et à la diffusion de documents scientifiques de niveau recherche, publiés ou non, émanant des établissements d'enseignement et de recherche français ou étrangers, des laboratoires publics ou privés. 


\title{
Revisiting Galvanomagnetic Effects in Conducting Ferromagnets Through Electromagnetic Field Angular Momentum Density and Magnetic Moment Coupling
}

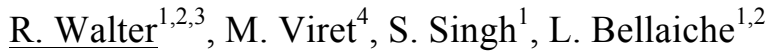 \\ ${ }^{1}$ Physics Department, University of Arkansas, Fayetteville, Arkansas 72701, USA \\ ${ }^{2}$ Institute for Nanoscience and Engineering, University of Arkansas, Fayetteville, Arkansas 72701, USA \\ ${ }^{3}$ Department of Mathematical Sciences, University of Arkansas, Fayetteville, Arkansas 72701, USA \\ ${ }^{4}$ Service de Physique de l'Etat Condense, CEA Saclay, DSM/IRAMIS/SPEC, URA CNRS 2464, 91191 Gif-Sur- \\ Yvette Cedex, France
}

This poster presentation discusses the recently proposed coupling between electromagnetic field angular momentum density and magnetic moments [1], with an overview of the different physical phenomena readily explained by this coupling [1-4] and emphasis on various galvanomagnetic effects [2] - anisotropic magnetoresistance and the planar Hall effect.

It is known that reversing the vector resulting from the cross product of electric and magnetic fields reverses the sense of rotation of magnetic vortices, through the coupling of the magnetic toroidal moment with this cross product [5-4]. By appropriately transforming this coupling energy, Ref. [1] demonstrated that the electromagnetic field angular momentum density can directly couple with magnetic moments to produce a physical energy. In that setting, this coupling reproduced the spin-current model in multiferroics and characterized a novel antiferroelectricity-driven magnetic anisotropy [1]. In conducting ferromagnets, it can explain the intrinsic anomalous Hall effect (AHE) and predict a novel Hall effect [3] as well as explain the anisotropic magnetoresistance (AMR) and the planar Hall effect (PHE) [2]. This energy also led to the prediction of novel spintronic effects [4] that bear resemblance to the so-called inverse Rashba-Edelstein effect. It may also be applicable to additional magneto-optical and spintronic phenomena.

Except for the phenomena discussed in Refs. [1] and [4], the proposed coupling has derived these effects according to a simple common method. The relevant electric and magnetic fields are first identified: for galvanomagnetic effects [2], an applied homogeneous electric field is used for the electric field and the Weiss field (proportional to magnetization) is used for the (homogeneous) magnetic field [9]. The coupling energy of a single conduction electron is taken as proportional to minus the scalar product of the field angular momentum and the magnetic moment of the electron, with some material dependent coupling constant as the proportionality coefficient. In this treatment of AMR and PHE, we are seeking anisotropy in longitudinal and transverse resistivity, so the electric field may adopt general Cartesian components along the longitudinal and transverse directions. Derivation of the appropriate expressions for AHE, AMR, and PHE then follows the standard derivation of the Hall effect in a Drude model. The equation of motion including the Lorentz force and the coupling force is considered component-wise in steady state; multiplying by the appropriate factor and summing over all electrons allows the component-wise equations to be expressed in terms of magnetization and current density. In steady state and with appropriate boundary conditions, the transverse applied electric field components cancel out the transverse current density, leaving only the longitudinal component of current density. Solving one of these resulting equations yields an expression for AHE. Solving for the longitudinal and transverse resistivities, the known expressions for AMR and PHE are recovered readily.

This derivation of galvanomagnetic effects has several advantages. One, as far as the authors are aware, it provides the first unified explanation for AHE together with AMR and PHE. Moreover, provided the material dependent coupling constant and the proportionality coefficient between the Weiss field and magnetization are each linear in spin-orbit interaction, our model reproduces the respective first- and second-order dependencies of AHE and AMR on spin-orbit interaction. Second, since in our model the difference between the resistivities for magnetization parallel and perpendicular to the current is proportional to the material dependent coupling constant; if this constant is negative in some materials, the phenomenon of so-called negative AMR naturally 
results. Third, the derivation for AHE worked as well in semiclassical electron dynamics and this allowed the coupling constant there to be expressed in terms of Berry-phase curvature [3]; if this can be shown to work for AMR, then a novel Berry-phase curvature theory of AMR may be possible. Fourth, if materials can be engineered to achieve a particular coupling constant, this could lead to design of novel electronic devices.

We thank C Paillard for valuable discussions. R Walter acknowledges this material is based upon work supported by the National Science Foundation Graduate Research Fellowship Program under Grant No. DGE0957325. Furthermore he acknowledges support from a University of Arkansas Distinguished Doctoral Fellowship in Mathematics and Physics. This work is financially supported by ARO Grant W911NF-12-1-0085.

\section{References}

[1] A. Raeliarijaona, S. Singh, H. Fu, and L. Bellaiche, Phys. Rev. Lett. 110, 137205 (2013).

[2] R. Walter, M. Viret, Surendra Singh and L. Bellaiche, J. Phys.: Condens. Matter 26, 432201 (2014).

[3] L. Bellaiche, Wei Ren and Surendra Singh, Phys. Rev. B 88, 161102(R) (2013).

[4] S. Bhattacharjee, S. Singh, D. Wang, M. Viret and L. Bellaiche, J. Phys.: Condens. Matter 26, 315008 (2014).

[5] H. Schmid, Ferroelectrics 252, 41 (2001).

[6] C. Ederer and N.A. Spaldin, Phys. Rev. B 76, 214404 (2007).

[7] V. M. Dubovik and V. V. Tugushev, Physics Reports 187, 145 (1990).

[8] A. A. Gorbatsevich, Yu.V. Kopaev, and V.V. Tugushev, Zh. Eksp. Teor. Fiz. 85, 1107 (1983) [Sov. Phys. JETP 58, 643 (1983)].

[9] NW Ashcroft and ND Mermin, Solid State Physics, (Philadelphia, PA: Saunders 1976). 